REVISTA DE ESTUDIOS E INVESTIGACIÓN

EN PSICOLOGÍA Y EDUCACIÓN

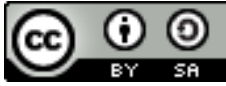

ISSN: 1138-1663; elSSN: 2386-7418

UDC / UMinho

2021, Vol. 8, No. 2, 229-241.

DOI: https://doi.org/10.17979/reipe.2021.8.2.8457

\title{
Relación entre ansiedad y rendimiento académico en alumnado de secundaria
}

\section{Relationship between anxiety and academic performance among secondary school students}

\begin{abstract}
Cecilia Colunga-Rodríguez (iD http://orcid.org/0000-0003-0328-788X Instituto Mexicano del Seguro Social: http//www.imss.gob.mx \& Centro Universitario de Ciencias de la Salud, Universidad de Guadalajara: https://www.cucs.udg.mx

Mario Ángel-González (D) https://orcid.org/0000-0002-5762-5170

Universidad de Guadalajara, Centro Universitario de Tonalá: http//www.cutonala.udg.mx Julio César Vázquez-Colunga (iD) https://orcid.org/0000-0001-8393-2505

Universidad de Guadalajara, Centro Universitario de Tonalá: http//www.cutonala.udg.mx Claudia Liliana Vázquez-Juárez (D) http://orcid.org/0000-0002-0932-0110 Universidad de Guadalajara, Centro Universitario de Tonalá: http//www.cutonala.udg.mx Bertha Alicia Colunga-Rodríguez (iD) https://orcid.org/0000-0002-2576-9225 Escuela Normal Superior de Jalisco: https://www.ensj.edu.mx
\end{abstract}

Guadalajara, Jalisco - México

Correspondencia relativa a este artículo Julio César Vázquez-Colunga: julio.vcolunga@academicos.udg.mx 


\title{
Resumen
}

El objetivo de este estudio fue indagar la relación entre ansiedad y rendimiento académico en estudiantes de secundaria. El diseño de la investigación fue transversal y correlacional, con una muestra probabilística de 156 estudiantes seleccionados aleatoriamente. Los datos se recogieron con la Escala de Ansiedad Manifiesta en Niños (CMAS-R) y un cuestionario de datos sociodemográficos. El rendimiento académico se calculó en base al promedio de calificaciones. La muestra tenía el mismo número de mujeres y varones, con una media de edad de 13.10 años ( $D E=0.94$ ); $69.23 \%$ cursaban primer año de secundaria, $19.23 \%$ segundo año y $11.54 \%$ tercero, y todos procedían de la zona metropolitana de Guadalajara (México). Los resultados mostraron indicios de ansiedad entre el $28.8 \%$ de los estudiantes. La media de calificación académica era 8.6 $(D E=0.82)$; el $42.3 \%$ tenía un rendimiento académico alto, el $38.5 \%$ medio y el $19.2 \%$ bajo. Se observó una puntuación media de ansiedad más alta entre los estudiantes con un rendimiento bajo, en comparación con los que tenían un rendimiento medio o alto. Al comparar los grupos de estudiantes con diferente rendimiento con la presencia o no de ansiedad, se encontró una correlación estadísticamente significativa. La relación entre mayor ansiedad y menor rendimiento académico sugiere la necesidad de evaluar periódicamente tanto la ansiedad como otros trastornos psicoafectivos entre los estudiantes mediante la vigilancia psicoepidemiológica, conjuntamente con el rendimiento escolar, con el fin de poner en marcha estrategias de actuación preventivas y correctoras.

Palabras clave: ansiedad, rendimiento académico, adolescentes, educación secundaria

\begin{abstract}
The aim of the study was to investigate the relationship between anxiety and academic performance among secondary school students. The research design was crosssectional and correlational, with a probability sample of 156 randomly selected students. The data for the study were collected using the Children's Manifest Anxiety Scale (CMAS-R) and a sociodemographic data questionnaire. Academic performance was calculated based on the students' grade average. The sample had the same number male and female students, with an average age of 13.10 years $(S D=0.94): 69.23 \%$ from first year, $19.23 \%$ from second year and $11.54 \%$ from third year, all from the metropolitan area of Guadalajara in Mexico. The results found indications of anxiety among $28.8 \%$ of the students. The average academic grade was 8.6 (SD $=.82$ ): $42.3 \%$ high academic performance, $38.5 \%$ medium performance and $19.2 \%$ low performance. A higher average anxiety score was observed among students with a low performance, compared to those with a medium or high performance. When comparing the groups of students with different academic performance with the presence or absence of anxiety, a statistically significant correlation was found. The correlation between greater anxiety and lower academic performance suggests the need for periodic assessment of both anxiety and other psycho-affective disorders among students through psychoepidemiological surveillance, in conjunction with school performance, in order to put in place preventive and remedial action strategies.
\end{abstract}

Keywords: anxiety, academic performance, adolescents, secondary education 
La Organización Mundial de la Salud (OMS) refiere que, a nivel mundial, entre el $10 \%$ y el 20\% de los adolescentes experimentan trastornos mentales y estos aparecen antes de los 14 años de edad, pero no se diagnostican ni se tratan adecuadamente (Organización Mundial de la Salud, 2020). A nivel mundial, la ansiedad ocupa el noveno lugar como enfermedad mental más frecuente entre los adolescentes de 15 a 19 años y el sexto sitio entre los de 10 a 14 años. La American Academy of Pediatrics ha reportado un incremento alarmante de ansiedad en adolescentes, de hasta el 20\% en el periodo comprendido del año 2007 al 2012 (McCarthy, 2019).

Según datos de la Encuesta Nacional de Epidemiología Psiquiátrica en México (ENEP), al menos $14.3 \%$ de la población, padece trastornos de ansiedad generalizada, considerada como el trastorno mental más común en el país, seguido de depresión y adicciones con un $9 \%$ cada una (Medina-Mora et al., 2003), sin embargo, no hay cifras oficiales actualizadas. En cuanto a los trastornos de ansiedad, la mitad de ellos se presentan antes de los 25 años, afectando por lo general, a adultos jóvenes y adolescentes (Medina-Mora et al., 2003). En los estudiantes de secundaria la prevalencia de ansiedad es del 18\%, siendo uno de los trastornos más comunes, junto con la depresión (Marín, 2015).

En una investigación que se realizó con 307 estudiantes de tres escuelas secundarias en una ciudad de Yucatán, se encontró que el $15.4 \%$ de los participantes presentaron sintomatología de ansiedad social (Marín-Ramírez, et al., 2015). En otro estudio en el que participaron 709 estudiantes universitarios mexicanos y el cual se llevó a cabo durante la pandemia por COVID 19 (mayo del 2020), se observó que el $37.7 \%$ de los participantes presentaron niveles altos de ansiedad (Pérez-Aranda et al., 2021), la diferencia en los porcentajes de estudiantes con ansiedad puede ser explicada por las presiones académicas debido al nivel educativo y por la situación de confinamiento ocasionada por la pandemia.

La ansiedad se caracteriza por la presencia de preocupaciones, temores intensos y continuos ante situaciones cotidianas. Los niños y los adolescentes con trastorno de ansiedad generalizada a menudo se preocupan excesivamente sobre su rendimiento en la escuela o en los deportes y suelen tener pensamientos catastróficos, produciendo síntomas físicos que dificultan el funcionamiento en la vida diaria (National Institute of Mental Health, 2017).

Si la ansiedad no se detecta oportunamente y se otorga el tratamiento pertinente, puede volverse crónica con la consecuente disminución en la calidad de vida del adolescente, así como el incremento en el riesgo de consumo de sustancias psicoactivas y de la comorbilidad con otros trastornos mentales (Marín, 2015; Riveros y Vinaccia-Alpi, 2017). 
Algunas investigaciones han correlacionado la presencia de ansiedad con el rendimiento académico. Una investigación en la que se compararon variables como ansiedad-estado (patrón transitorio de emociones), ansiedad-rasgo (patrón relativamente constante de emociones) y estrategias de aprendizaje, se observaron diferencias estadísticamente significativas $(F=9.17$, $p<0.05)$ entre el grupo de alto y bajo rendimiento académico solo para la variable ansiedadrasgo (Martínez-Álvarez y Lajo, 2018).

En otro estudio realizado con 535 estudiantes mexicanos de secundaria (González et al., 2018), se encontraron correlaciones estadísticamente significativas entre las variables asertividad y ansiedad $(r=.182, p<.01)$, entre rendimiento académico y asertividad $(r=-.203$, $p<.01)$, y entre rendimiento académico y el componente preocupaciones sociales de la escala de ansiedad manifiesta en niños $(r=-.124, p<.01)$, se puede decir que el grado de asertividad de los estudiantes puede regular los niveles de ansiedad e impactar en el desempeño académico. También se ha estudiado el impacto de la ansiedad que producen asignaturas específicas como las matemáticas en el rendimiento académico, en un estudio realizado con 127 estudiantes de secundaria de una escuela pública en Colombia, se observó que existe una correlación negativa significativa entre ansiedad matemática y rendimiento académico en matemáticas ( $r=-.247, p<.05)$, es decir a mayor ansiedad menor rendimiento académico, sin embargo los niveles de ansiedad presentados fueron bajo (Villamizar et al., 2020)

Otros autores han referido distintos factores implicados en el rendimiento académico, por ejemplo, estados emocionales, el funcionamiento cognitivo y el índice de masa corporal (ÁngelGonzález et al., 2020; Martelo y Arévalo, 2017). Por su lado, se ha estudiado el impacto de la ansiedad en relación a diferentes variables, por ejemplo, el desempeño deportivo, la autoestima y la comorbilidad con otros trastornos mentales (Friesen y Markowsky, 2021; Hernández et al., 2018; Núñez y Garcia, 2017).

Quienes han investigado el rendimiento académico, se han encontrado con la complejidad que implica definir y evaluar dicho concepto (Lamas, 2015). Para esta investigación hemos decidido medir el rendimiento académico por medio de las calificaciones de los estudiantes participantes.

En México son pocos los estudios recientes que han reportado resultados en población de adolescente que estudian la secundaria, estableciendo la relación entre ansiedad y rendimiento académico (González et al., 2018; Martínez-Álvarez y Lajo, 2018). Debido a la poca investigación realizada en este campo y al impacto que tiene la presencia de ansiedad en el proceso de aprendizaje de los estudiantes, esta investigación es pertinente para conocer la 
situación en la que se encuentra los estudiantes de la secundaria donde se realizó es estudio y contar con datos empíricos que se puedan considerar e incluir en los programas de tutoría.

El objetivo de esta investigación fue describir si existe una correlación entre el rendimiento académico y la presencia de ansiedad en un grupo de estudiantes de secundaria. Es importante mencionar que esta investigación formó parte de un proyecto donde se estudiaron diversas variables (psicológicas, fisiológicas y socioeconómicas) y su asociación con el rendimiento académico.

\section{Método}

\section{Participantes}

Se seleccionó una escuela secundaria pública de la Zona Metropolitana de Guadalajara en Jalisco, México. La población diana incluyó 156 estudiantes (50\% mujeres y 50\% varones) de entre 12 y 15 años de edad $(M=13.10, D E=0.94)$, el 69.23\% cursaba el primer grado de secundaria, el $19.23 \%$ el segundo grado y el $11.54 \%$ el tercer grado.

El tamaño de la muestra se calculó tomando en cuenta la población total de estudiantes inscritos en el plantel educativo al momento de realizar la investigación, también se consideró una prevalencia esperada de ansiedad de $18 \%$, de acuerdo a lo reportado en la literatura para población de adolescentes mexicanos (Marín, 2015). Se determinó un 95\% de confianza y 5\% de error. El muestreo fue probabilístico, para lo cual se utilizó una tabla de números aleatorios. Se seleccionaron los estudiantes de acuerdo al número que se le otorga en las listas de asistencias, comenzando por un grupo de primer año, un grupo de segundo y un grupo de tercero, volviendo en ese orden hasta obtener la totalidad de participantes calculado para la muestra. Si un estudiante seleccionado se encontraba ausente, se sustituía por el que lo seguía en la lista de asistencia.

Se consideró como criterio de inclusión a todo estudiante que estuviera inscrito en calidad de alumno regular en el momento de aplicar los instrumentos, que deseara participar en el estudio.

El diseño fue transversal analítico y correlacional.

\section{Instrumentos}

Escala de Ansiedad Manifiesta en Niños CMAS-R (Reynolds y Richmond, 1997). Se trata de un instrumento de autoinforme, que fue diseñado para evaluar el nivel y la naturaleza de la ansiedad de niños y adolescentes con edades de entre 6 y 19 años. El instrumento está validado 
en población mexicana y tiene un coeficiente de fiabilidad (alfa de Cronbach) de .74. La escala contiene 37 reactivos de respuesta dicotómica, divididos en tres dimensiones: i) Ansiedad fisiológica, se refiere a manifestaciones fisiológicas de ansiedad, como dificultades del sueño, náusea y fatiga; ii) Inquietud/hipersensibilidad, se refiere a la preocupación obsesiva acerca de una variedad de cosas percibidas por el menor y a temores de daño o aislamiento emocional; iii) Preocupaciones sociales/concentración, se refiere a pensamientos distractores y miedo social o interpersonal, expresan una preocupación por la autocomparación con otras personas, que llevan a deficiencias en la concentración y la atención.

Cédula de Datos Sociodemográficos: Cuestionario desarrollado ad hoc para registrar los datos como sexo, edad, grado escolar y calificación promedio de cada uno de los participantes. El criterio que se consideró para establecer los niveles del rendimiento escolar fue: bajo = calificación de 5 a 6, medio = calificación de 7 a 8, alto = calificación de 9 a 10 .

\section{Procedimiento}

Inicialmente, se concertó una reunión con el director del plantel educativo, se le explicaron los objetivos de la investigación y se solicitó su autorización para realizarla. Después, se envió un comunicado a los padres de los estudiantes, explicando el propósito del estudio y se solicitó que firmaran el consentimiento informado, en caso de que permitieran que sus hijos participaran.

El día de la aplicación de los instrumentos se informó a los estudiantes de la importancia de su participación voluntaria y se les explicó que estaban en la libertad de dejar de participar en el momento que quisieran, sin ninguna repercusión de ningún tipo y que esta negativa podría decidirse independientemente de que sus padres si hubiesen aceptado y firmado el consentimiento. A la vez, se les aclaró que no habría ningún tipo de incentivo por participar. Una vez que manifestaron verbalmente su aceptación, se comenzó con la aplicación de los instrumentos.

La recogida de datos fue realizada por personal capacitado en la aplicación de pruebas psicométricas.

\section{Aspectos éticos}

Este estudio consideró los principios éticos de la declaración de Helsinki (Asociación Médica Mundial, 2013) en el apartado correspondiente a investigación en seres humanos. Esta investigación se clasifica como de riesgo mínimo. Se aplicó un consentimiento basado en la Ley General de Salud (2015) de los Estados Unidos Mexicanos en su capítulo quinto "Investigación 
para la salud", artículo 100. Inicialmente, los padres otorgaron el consentimiento informado firmando una carta, posteriormente, cada estudiante aceptó participar voluntariamente en la investigación para lo cual se les solicitó su asentamiento verbal. Cabe señalar que el protocolo de investigación, fue aprobado por el comité universitario de ética e investigación. Al finalizar el estudio se entregó al director del plantel educativo un reporte de los resultados, considerando el anonimato de los alumnos, con excepción de los casos que por la severidad de la ansiedad se requirió notificar a los padres de familia y maestros para su oportuna atención.

\section{Análisis estadístico}

Se aplicó estadística descriptiva y pruebas de normalidad mediante Kolmogorov-Smirnov. Para medir la relación entre el rendimiento escolar con la presencia de ansiedad, se utilizó la prueba Ji al cuadrado. Además, se utilizó el coeficiente de contingencia para medir la intensidad de la relación entre las variables de rendimiento académico y ansiedad. Se consideraron estadísticamente significativos los resultados con valores de $p<.05$. El análisis estadístico se realizó con el programa IBM SPSS v. 25 para Windows.

\section{Resultados}

En la Tabla 1 se muestran las puntuaciones de ansiedad por dimensiones del instrumento CMAS-R, según nivel de rendimiento académico. Se encontró que los estudiantes con rendimiento bajo presentaron promedios más altos en las puntuaciones de ansiedad, para cada una de las dimensiones, así como en la puntuación total.

\section{Tabla 1}

Ansiedad y nivel de rendimiento académico $(N=156)$

\begin{tabular}{lccccccccc}
\hline & \multicolumn{4}{c}{ Bajo $(n=30)$} & \multicolumn{4}{c}{ Rendimiento académico } & \multicolumn{3}{c}{ Medio $(n=60)$} & \multicolumn{3}{c}{ Alto $(n=66)$} \\
& Min/Máx. & $M$ & $D E$ & Min/máx. & $M$ & $D E$ & Min/Máx. & $M$ & $D E$ \\
\hline Dimensiones del CMAS-R & $6 / 19$ & 11.5 & 3.3 & $5 / 17$ & 9.9 & 3.0 & $5 / 15$ & 9.5 & 2.9 \\
\hline Ansiedad fisiológica & $5 / 15$ & 11.0 & 3.1 & $7 / 15$ & 10.3 & 2.1 & $4 / 16$ & 10.1 & 3.0 \\
Inquietud/hipersensibilidad & $5 / 16$ & 10.9 & 3.1 & $5 / 15$ & 9.2 & 3.0 & $5 / 16$ & 9.4 & 2.8 \\
$\begin{array}{l}\text { Preocupaciones sociales } \\
\text { /concentración }\end{array}$ & & & & & & & & & \\
Total & $32 / 71$ & 55.5 & 12.0 & $41 / 74$ & 51.3 & 9.6 & $27 / 71$ & 50.6 & 10.9 \\
\hline
\end{tabular}

Por otra parte, en la Tabla 2 se observa que 45 estudiantes de la muestra (28.8\%) presentaron ansiedad y 111 (71.2\%) no.

Al comparar los grupos de estudiantes con rendimiento académico bajo, medio y alto con la presencia o no de ansiedad, se encontró que existe una asociación estadísticamente 
significativa entre grupos $\left(X i^{2}(2)=8.9, p<.05\right)$, además se obtuvo un coeficiente de contingencia de .23, que denota una baja correlación entre las variables de rendimiento académico y presencia de ansiedad.

\section{Tabla 2}

Comparación de rendimiento académico en función de la presencia de ansiedad

\begin{tabular}{lccc}
\hline Rendimiento académico & Sin ansiedad & Con ansiedad & Total \\
& $f(\%)$ & $f(\%)$ & $f(\%)$ \\
\hline Bajo & $15(9.6)$ & $15(9.6)$ & $30(19.2)$ \\
Medio & $48(30.8)$ & $12(7.7)$ & $60(38.5)$ \\
Alto & $48(30.8)$ & $18(11.5)$ & $66(42.3)$ \\
Total & $111(71.2)$ & $45(28.8)$ & $156(100.0)$ \\
\hline
\end{tabular}

\section{Discusión}

Este estudio realizado con estudiantes de una secundaria pública del municipio de Tlaquepaque, Jalisco en México, mostró que $28.8 \%$ de los estudiantes tienen ansiedad. La media de calificación fue $8.6(D E=.82)$, el $42.3 \%$ tenía un rendimiento académico alto, el $38.5 \%$ un rendimiento medio y el $19.2 \%$ un rendimiento bajo. Los datos obtenidos en torno al rendimiento académico se obtuvieron del registro de calificaciones y no se contó con otros recursos que permitieran detallar otros aspectos que otras investigaciones si han incluido, por ejemplo, realizar comparaciones de acuerdo a materias específicas, como matemáticas, español o inglés (LeónJacinto, 2016; Rabadán y Orgambídez, 2018; Salazar-Rendón et al., 2018; Villamizar et al., 2020).

El problema de la ansiedad representa una problemática que afecta seriamente el desarrollo integral y la calidad de vida de los adolescentes (Riveros y Vinaccia-Alpi, 2017). En este estudio, la prevalencia de ansiedad en la muestra estudiada, fue superior al promedio reportado por la OMS para la población de adolescentes de entre 10 y 14 años (Organización Mundial de la Salud, 2020), y aunque no se incluyeron variables mediadoras (estrategias de aprendizaje, asertividad, funcionamiento cognitivo y estrategias de autorregulación) como lo investigado por otros autores (Barreto-Trujillo y Álvarez-Bermúdez, 2020; González et al., 2018; Martelo y Arévalo, 2017; Martínez-Álvarez y Lajo, 2018), se encontró una correlación estadísticamente significativa entre el rendimiento académico y la presencia de ansiedad, siendo los estudiantes con rendimiento académico bajo quienes obtuvieron mayores promedios de puntuación en todas las subescalas del instrumento CMAS-R, en contraste, en otro estudio se reportó una correlación entre rendimiento académico y solamente la subescala preocupación sociales del mismo instrumento (González et al., 2018). 
Como ya se describió en esta investigación, el estudio de la ansiedad es de gran importancia para comprender los niveles en el rendimiento académico, pero sus implicaciones pueden ser más complejas. En los resultados de un estudio se mostró que los estudiantes con niveles altos de ansiedad ante los deberes escolares tienen un perfil motivacional de evitación al fracaso, es decir, a mayor miedo al fracaso escolar mayor ansiedad, y este temor es considerado un factor motivacional (Regueiro et al., 2015). Otro estudio reportó que los estudiantes adolescentes con alteraciones del sueño (dormían menos que lo recomendado para su edad) tuvieron mayor ansiedad y mayores logros académicos en comparación con los estudiantes adolescentes con menos alteraciones del sueño (Zhang et al., 2021). Estas investigaciones han encontrado que la ansiedad puede ser un factor motivante para mejorar el rendimiento académico, en este sentido, el presente estudio revela la importancia de tener en cuenta la ansiedad escolar como un constructo multidimensional, incluso, debido a la etapa del desarrollo de la población en estudio, podría considerarse estudiar la influencia de la crianza y los estilos parentales en el desarrollo de ansiedad en adolescentes escolarizados (Mishra y Kiran, 2018; Xu et al., 2017).

Aunque el objetivo de esta investigación fue determinar la asociación entre las variables de estudio, también se considera importante que se realicen investigaciones con diseños prospectivos, con el fin de buscar una posible relación entre las variables tratadas en este trabajo, ya que no se ha realizado un abordaje profundo de cómo un fenómeno como la ansiedad se asocia con el rendimiento académico en adolescentes mexicanos.

Esta investigación presenta varias limitaciones. En primer lugar, no es posible establecer relaciones causales ni determinar la influencia de la ansiedad sobre el rendimiento académico. En segundo lugar, es necesario aumentar el tamaño de la muestra e incorporar escuelas secundarias de distintas zonas de la ciudad. En tercer lugar, debido a que el rendimiento académico en este estudio, se representa a través de la ponderación numérica en función del cumplimiento de los criterios que los programas escolares establecen tales como: elaboración de productos específicos, asistencia a clase, participación, entre otros, sería necesario establecer otros parámetros que contemplen pruebas escritas y ejecutivas a los alumnos para evaluar su desempeño ante la exigencia escolar.

A pesar de las limitaciones detectadas, este estudio presenta como fortaleza, el hecho de ser uno de los pocos estudios actuales realizados en México sobre esta problemática. Desde un punto de vista práctico, los resultados sugieren realizar diseños de investigación específicos para estudiar poblaciones similares, no sólo con el propósito de detectar la prevalencia de la ansiedad 
y otros trastornos comunes en la adolescencia, como la depresión y consumo de drogas, sino para proponer líneas de acción concretas, de cara a disminuir el problema de la salud mental y el bajo rendimiento académico.

Los resultados permiten responder a la pregunta de investigación, al afirmar que existen diferencias en el rendimiento académico de los estudiantes de secundaria con ansiedad, contra los que no la presentan. Como parte de los principales hallazgos, podemos concluir, por un lado, que la prevalencia de ansiedad fue más alta que la reportada a nivel nacional para población adolescente, en una proporción de casi 3 de cada 10 estudiantes; el rendimiento académico en 4 de cada 10 alumnos es alto, existiendo diferencia de significancia estadística entre los grupos, clasificados por la presencia de ansiedad.

Por otro lado, el promedio de calificaciones más altas, la presentan los estudiantes sin ansiedad, aunque la dependencia entre las dos variables principales de estudio es débil. Debido al diseño del estudio, no se pueden establecer relaciones causales entre variables, razón por la cual, se requiere realizar estudios con otros diseños metodológicos, para poder determinar la posible existencia de relación causal y variables predictivas, entre ansiedad y rendimiento académico.

Los resultados de esta investigación ponen de manifiesto, que los estados emocionales comunes en los estudiantes de secundaria como puede ser la ansiedad, ya sea que se encuentren vinculados a su desarrollo madurativo biológico o tenga causas externas de tipo social, pueden influir en su rendimiento académico, y con ello, afectar el pleno desarrollo de su personalidad y de sus potencialidades, aspecto que es considerado como uno de los derechos humanos.

Por lo tanto, los hallazgos deben tomarse en cuenta para realizar acciones preventivas a nivel primario (promoción de la salud y educación para la salud), secundario (detección oportuna y limitación del daño); y terciario (Rehabilitación y readaptación), esto implica un trabajo a nivel de la institución por medio de la capacitación del personal escolar, de los grupos, tanto de estudiantes, como de padres de familia y a nivel individual, para quienes ya presentan la problemática del trastorno, para saber cómo se puede actuar en estas situaciones, incluso por medio de guías o protocolos de actuación, para actuar adecuada y oportunamente.

El establecimiento de un sistema interno de vigilancia psicoepidemiológica, también serviría para llevar un seguimiento e identificar el comportamiento de la ansiedad y su relación con el rendimiento académico, pudiendo incluir otros trastornos comunes en adolescentes como puede ser la ansiedad, la dinámica familiar y el apoyo social que puede representar la escuela.

Agradecimiento: A las autoridades de la escuela secundaria donde se realizó esta investigación, por el apoyo brindado. 


\section{Referencias}

ÁNGEL-GONZÁLEZ, Mario; VÁZQUEZ-JUÁREZ, Claudia Liliana; COLUNGA-RODRÍGUEZ, Bertha Alicia; DÁVALOS-PICAZO, Gabriel; VÁZQUEZ-COLUNGA, Julio César; HERNÁNDEZCORONA, Diana Mercedes; HERNÁNDEZ-BITARD, Deborah Georgette; \& COLUNGARODRÍGUEZ, Cecilia (2020). Estudio transversal para comparar el rendimiento académico en estudiantes mexicanos de educación secundaria en función de su índice de masa corporal. Revista Española de Nutrición Humana y Dietética, 24(3), 218-225. https://doi. org/10.14306/renhyd.24.3.987

Asociación Médica Mundial (2013). Declaración de Helsinki de la AMM - Principios éticos para las investigaciones médicas en seres humanos. Disponible en: https://www.wma.net/ es/policies-post/declaracion-de-helsinki-de-la-amm-principios-eticos-para-lasinvestigaciones-medicas-en-seres-humanos/

BARRETO-TRUJILLO, Francisco Javier; \& ÁLVAREZ-BERMÚDEZ, Javier (2020). Estrategias de autorregulación del aprendizaje y rendimiento académico en estudiantes de bachillerato. Revista de Estudios e Investigación en Psicología y Educación 7(2), 184-193. https://doi.org/10.17979/reipe.2020.7.2.6570

FRIESEN, Kelsey; \& MARKOWSKY, Ashley (2021). The diagnosis and management of anxiety in adolescents with comorbid ADHD. The Journal for Nurse Practitioners, 17, 65e69. https://doi.org/10.1016/j.nurpra.2020.08.014

GONZÁLEZ, Claudia; GUEVARA, Yolanda; JIMÉNEZ, David; \& ALCÁZAR, Raúl J. (2018). Relación entre Asertividad, Rendimiento Académico y Ansiedad en una Muestra de Estudiantes Mexicanos de Secundaria. Acta colombiana de Psicología, 21(1), 116-127. https://doi.org/ 10.14718/ACP.2018.21.1.6

HERNÁNDEZ, M. Á Ángeles; BELMONTE, Lorena; \& MARTíNEZ, M.a de las Mercedes (2018). Autoestima y ansiedad en los adolescentes. REIDOCREA, 7, 269-278. https://doi.org/10. 30827/Digibug.54133

LAMAS, Héctor (2015). Sobre el rendimiento escolar. Propósitos y Representaciones, 3(1), 313386. https://doi.org/10.20511/pyr2015.v3n1.74

LEÓN-JACINTO, Delia (2016). Ansiedad escolar, Autoestima y aprendizaje matemático en estudiantes de primaria. [Tesis Doctoral, Universidad César Vallejo, Perú]. Repositorio Digital Institucional: https://hdl.handle.net/20.500.12692/22445

Ley General de Salud (2015). Cámara de diputados del H. Congreso de la Unión. Secretaría General. Secretaría de Servicios Parlamentarios. Disponible en: http://www.diputados. gob.mx/LeyesBiblio/ref/lgs.htm 
MCCARTHY, Claire (2019). La ansiedad en los adolescentes va en aumento: ¿qué está pasando? American Academy of Pediatrics: https://www.healthychildren.org/Spanish/healthissues/conditions/emotional-problems/Paginas/Anxiety-Disorders.aspx

MARÍN, María (2015). Estrés en estudiantes de educación superior de Ciencias de la Salud. Revista Iberoamericana para la Investigación y el Desarrollo Educativo, 6(11), 675-687. https://www.ride.org.mx/index.php/RIDE/article/view/145

MARÍN-RAMIIREZ, ALONSO HUMBERTO; MARTÍNEZ-DÍAZ, German Jesús; \& ÁVILA-AVILÉS, José Manuel (2015). Detección de sintomatología de ansiedad social y factores asociados en adolescentes de Motul, Yucatán, México. Revista Biomédica, 26(1), 23-31. https://doi. org/10.32776/revbiomed.v26i1.5

MARTELO, Okendy Melissa; \& ARÉVALO, Jessica Marcela (2017). Funcionamiento cognitivo y estados emocionales de un grupo de niños y adolescentes con bajo rendimiento académico. Neuropsicología Latinoamericana, 9(3), 13-22. https://www.neuropsico latina.org/index.php/Neuropsicologia_Latinoamericana/article/view/383

MARTÍNEZ-ÁLVAREZ, Isabel; \& LAJO, Alicia (2018). Estudio neuropsicológico de la funcionalidad visual, las estrategias de aprendizaje y la ansiedad en el rendimiento académico. Aula Abierta, 47(2), 245-254. https://doi.org/10.17811/rifie.47.2.2018.245-254

MEDINA-MORA, María Elena; BORGES, Guilherme; LARA, Carmen; BENJET, Corina; BLANCO, Jerónimo; FLEIZ, Clara; VILLATORO, Jorge; ROJAS, Estela; ZAMBRANO, Joaquín; CASANOVA, Leticia; \& AGUILAR-GAXIOLA, Sergio (2003). Prevalencia de trastornos mentales y uso de servicios: Resultados de la encuesta nacional de Epidemiología Psiquiátrica en México. Salud Mental, 26(4), 1-16. http://www.revistasaludmental.mx/ index.php/salud_mental/article/view/956

MISHRA, Pinki; \& KIRAN, U.V (2018). Parenting style and social anxiety among adolescents. International Journal of Applied Home Science, 5(1), 117-123.

National Institute of Mental Health (2017). Trastorno de ansiedad generalizada: cuando no se pueden controlar las preocupaciones. National Institute of Mental Health (NIH): https://www.nimh.nih.gov/health/publications/espanol/trastorno-de-ansiedadgeneralizada-cuando-no-se-pueden-controlar-las-preocupaciones-new

NÚÑEZ, Antonio; \& GARCIA, Alejandro (2017). Relación entre el rendimiento y la ansiedad en el deporte: una revisión sistemática. Retos, 32, 172-177. https://doi.org/10.47197/retos. v0i32.53297

Organización Mundial de la Salud (2020). Salud mental del adolescente. OMS. https://www.who. int/es/news-room/fact-sheets/detail/adolescent-mental-health

PÉREZ-ARANDA, Gabriela Isabel; ESTRADA-CARMONA, Sinuhe; \& CATZIN-LÓPEZ, Eric Alejandro (2021). Confinamiento y ansiedad en estudiantes universitarios del sureste mexicano 
durante la epidemia de COVID-19. Comunidad y Salud, 19(1), 25-32. Disponible en: http://servicio.bc.uc.edu.ve/fcs/index1.htm

RABADÁN, Mercedes; \& ORGAMBÍDEZ, Alejandro (2018). Ansiedad idiomática en español como lengua extranjera y rendimiento académico en la enseñanza superior. Revista de Estudios e Investigación en Psicología y Educación, 5(1), 29-35. https://doi.org/10.17979/reipe. 2018.5.1.2905

REYNOLDS, Cecil; \& RICHMOND, Bert (1997). Escala de ansiedad manifiesta en niños (revisada). Manual Moderno.

REGUEIRO, Bibiana; RODRÍGUEZ, Susana; PIÑEIRO, Isabel; FREIRE, Carlos; FERRADÁS, Mar; GAYO, Elena; \& VALLE, Antonio (2015). Perfiles motivacionales, implicación y ansiedad ante los deberes escolares y rendimiento académico en estudiantes de Educación Secundaria Obligatoria. Revista de Estudios e Investigación en Psicología y Educación, Extr.(01), 093-096. https://doi.org/10.17979/reipe.2015.0.01.469

RIVEROS, Fernando; \& VINACCIA-ALPI, Stefano (2017). Relación entre variables sociodemográficas, patogénicas y salutogénicas, con la calidad de vida en estudiantes universitarios colombianos. Revista de Psicopatología y Psicología Clínica, 22, 229-241. https://doi.org/ 10.5944/rppc.vol.22.num.3.2017.16330

SALAZAR-RENDÓN, Juan Carlos; MÉNDEZ, Nina; \& AZCORRA, Hugo (2018). Asociación entre el sobrepeso y la obesidad con el rendimiento académico en estudiantes de secundaria de la ciudad de Mérida, México. Boletín Médico Del Hospital Infantil de México, 75(2), 94103. https://doi.org/10.24875/bmhim.m18000018

VILLAMIZAR, Gustavo; ARAUJO, Tammi Yulien; \& TRUJILLO, Wenddy Jurany (2020). Relación entre ansiedad matemática y rendimiento académico en matemáticas en estudiantes de secundaria. Ciencias Psicológicas, 14(1), e-2174. https://doi.org/10.22235/cp.v14i1.2174

XU, Jihong; NI, Shiguang; RAN, Maosheng; \& ZHANG, Chengping (2017). The relationship between parenting styles and adolescents' social anxiety in migrant families: a study in Guangdong, China. Frontiers in Psychology, 8, 626. http://doi.org/10.3389/fpsyg.2017.00626

ZHANG, Xiaoning; DIMITRIOU, Dagmara; \& HALSTEAD, Elizabeth J. (2021). Sleep, anxiety, and academic performance: a study of adolescents from public high schools in China. Frontiers in Psychology, 12, 678839. http://doi.org/10.3389/fpsyg.2021.678839

Fecha de recepción: 18 de mayo de 2021.

Fecha de revisión: 23 de octubre de 2021. Fecha de aceptación: 26 de octubre de 2021. Fecha de publicación: 1 de diciembre de 2021. 